\title{
High-Performance All-Polymer Solar Cells and Photodetectors Enabled by a High-Mobility n-Type Polymer and Optimized Bulk-Heterojunction Morphology
}

Yanfei Zhao, ${ }^{\dagger,+}, \perp$ Tao Liu, ${ }^{\dagger, \perp}$ Baoqi Wu, ${ }^{\dagger, \perp}$ Song Zhang, ${ }^{\S}$ Nathaniel Prine, ${ }^{\S}$ Long

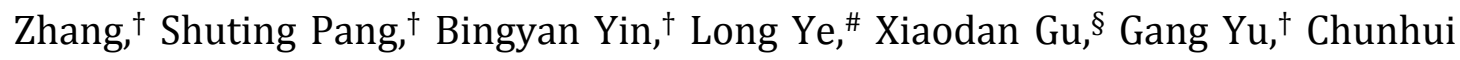
Duan, ${ }^{*},+, \|$ Fei Huang, ${ }^{\dagger}$ and Yong $\mathrm{Cao}^{\dagger}$

†Institute of Polymer Optoelectronic Materials and Devices, State Key Laboratory of Luminescent Materials and Devices, South China University of Technology, Guangzhou 510640, P. R. China

₹ School of Materials Science and Engineering, Dongguan University of Technology, Dongguan 523808, P. R. China

$\S$ School of Polymer Science and Engineering, University of Southern Mississippi, Hattiesburg, MS 39406, United States

\# School of Materials Science and Engineering, Tianjin University, Tianjin 300350, P. R. China "I State Key Laboratory of Fine Chemicals, Dalian University of Technology, Dalian 116024, P. R. China

\section{Experimental Section}

Materials: PNDI-DTBT was synthesized by following the procedure described in literature. ${ }^{1}$ Three batches of PNDI-DTBT with different molecular weights were synthesized to investigate the effect of molecular weight on the device performance. PBDB-T and PCE10 were purchased from Solarmer Materials Inc., and J51 was purchased form 1-Material Inc. The other chemicals and solvents 
were purchased from commercial sources (Sigma Aldrich, Acros, Stream, and Alfa Aesar, etc.) and used as received unless otherwise specified.

UV-vis-NIR absorption spectra: UV-vis-NIR spectra were recorded on a Shimadzu UV-3600 spectrophotometer at room temperature. All solutions were prepared in chlorobenzene (CB) with a concentration of $0.05 \mathrm{mg} \mathrm{mL}^{-1}$. Films were made by spin-coating the CB solutions onto glass substrates.

Square wave voltammetry (SWV): SWV is more sensitive than cyclic voltammograms (CV) to investigate electrochemical properties and frontier orbital energy levels of conjugated polymers and small molecules. ${ }^{2-5}$ The measurements were performed on a CHI660A electrochemical workstation in a solution of tetrabutylammonium hexafluorophosphate $\left(\mathrm{Bu}_{4} \mathrm{NPF}_{6}, 0.1 \mathrm{M}\right)$ in acetonitrile at a scan rate of $50 \mathrm{mV} \mathrm{s}^{-1}$. Indium tin oxide (ITO), a platinum wire and an $\mathrm{Ag} / \mathrm{AgCl}$ electrode were used as working electrode, counter electrode and reference electrode, respectively. The film samples were prepared by spin-coating on the surface of the working electrode. The highest occupied molecular orbital (HOMO) and lowest unoccupied molecular orbital (LUMO) energy levels can be calculated with reference to $\mathrm{Fc} / \mathrm{Fc}^{+}$as follows.

$$
\begin{aligned}
& E_{\text {НОМO }}=-\left(E_{\text {ox }}+5.13-E_{F C / F C}+\right) e V \\
& E_{\text {HOMO }}=-\left(E_{\text {red }}+5.13-E_{F C / F C^{+}}\right) \mathrm{eV}
\end{aligned}
$$

Fabrication and characterization of single-carrier devices: The charge carrier mobilities were measured by space-charge-limited current (SCLC) method based 
on single-carrier devices. The structures are ITO/PEDOT:PSS (40 nm)/active layer $(100 \mathrm{~nm}) / \mathrm{MoO}_{\mathrm{x}}(12 \mathrm{~nm}) / \mathrm{Ag}(90 \mathrm{~nm})$ for hole-only devices and ITO/ZnO (40 $\mathrm{nm}) /$ active layer $(100 \mathrm{~nm}) / \mathrm{PFN}-\mathrm{Br}(5 \mathrm{~nm}) / \mathrm{Ag}(100 \mathrm{~nm})$ for electron-only devices. The current density $(J)$-voltage $(V)$ characteristics at space-charge-limited-current region can be described by the Mott-Gurney law $J=(9 / 8) \varepsilon_{0} \varepsilon_{\mathrm{r}} \mu\left(\left(V^{2}\right) /\left(d^{3}\right)\right)$, where $J$ is the current, $\mu$ is the zero-field mobility, $\varepsilon_{0}$ is the permittivity of free space, $\varepsilon_{\mathrm{r}}$ is the relative permittivity of the material, $d$ is the thickness of the active layers, and $V$ is the effective voltage. $V$ can be calculated by $V=V_{\text {appl }}-V_{\mathrm{bi}}-V_{\mathrm{s}}$, where $V_{\mathrm{appl}}$ is applied voltage, $V_{\mathrm{bi}}$ is built-in voltage, $V_{\mathrm{s}}$ is the voltage drop from the series resistance of the whole device except for the active layers. The charge carrier mobility can be calculated by fitting the dark current from the slope of the $J^{1 / 2} \sim V$ curves.

Transient photovoltage (TPV) and transient photocurrent (TPC): The TPV measurement was conducted under the open-circuit condition by connecting the device to a Tektronix TDS 3052C digitizing oscilloscope. A small pulsed perturbation of excess charge carriers was produced by a weakened $620 \mathrm{~nm}$ laser pulse with a pulse width of 120 fs. The TPC measurement was performed under the short-circuit condition in dark by connecting the device to the ground through a small resistor. The decay life time $(\tau)$ was obtained by fitting a single exponential function to the transient photovoltage or transient photocurrent signal. 
Grazing incidence wide-angle X-ray scattering (GIWAXS): The GIWAXS measurements were performed on an in-house Xenocs Xeuss 2.0 beamline. The incident X-ray has a wavelength of $1.54 \AA$ and an incident angle of $0.2^{\circ}$. The sample is kept under vacuum to avoid air scattering with a sample-to-detector distance of $150 \mathrm{~mm}$. The data is collected by a Pilatus $1 \mathrm{M}$ detector and analyzed using Igor 8 software together with the Nika package and WAXSTools.

Transmission electron microscopy (TEM): Transmission electron microscopy images were collected from a JEM-2100F transmission electron microscope operated at $200 \mathrm{kV}$.

Nanoscale infrared-atom force microscopy (AFM-IR): Samples were measured using a nanoIR3 AFM-IR from Anasys Instruments (Santa Barbara, CA) coupled to a MIRcat-QT ${ }^{T M}$ quantum cascade, mid-infrared laser by Daylight Solutions (frequency range of $917-1700 \mathrm{~cm}^{-1}$ and $1900-2230 \mathrm{~cm}^{-1}$ and repetition rate of 1,470 kHz). AFM-IR data are collected in tapping mode using a gold-coated, AFM probe (spring constant (k): $40 \mathrm{~N} / \mathrm{m}$ and resonant frequency $\left(\mathrm{f}_{\mathrm{o}}\right): 300 \mathrm{kHz}$ ). The pulsed, mid-IR laser is tuned to frequencies unique to each component as determined by FTIR characterization. Acquired images are flattened using Analysis Studio software.

Resonant soft X-ray scattering (RSoXS): RSoXS measurements were performed at beamline 11.0.1.2 of Advanced Light Source. Thin film samples were spincoated on top of the PEDOT:PSS covered Si wafers under exactly the same 
condition as those for solar cell fabrication. Then BHJ thin films were floated and transferred onto silicon nitride membrane windows. The scattering was conducted in transmission mode and signals were collected under vacuum by Princeton Instrument PI-MTE CCD camera. Data analysis was conducted by Igor software with a modified Nika package.

X-ray photoelectron spectroscopy (XPS): XPS measurements of the samples (donor:acceptor/PEDOT:PSS/ITO) were performed on ESCALAB Xi+ (Thermo Fisher Scientific). The samples were sputtered with an $\mathrm{Ar}^{+}$gun at $2 \mathrm{kV} \times 3.3 \mu \mathrm{A}$ and etched from the air/active layer interface. The scanning area for XPS depth profile was $2.5 \mathrm{~mm} \times 2.5 \mathrm{~mm}$, and the composition data were acquired from an area equal to a circle with a diameter of $0.5 \mathrm{~mm}$.

Contact angle measurements: The contact angle tests were performed on a Dataphysics OCA40 Micro surface contact angle analyzer.

Flory-Huggins interaction parameters: The Flory-Huggins interaction parameters can be derived from contact angles as follows. ${ }^{6-9}$

1) Choose liquids 1 and 2 (whose polar and dispersive components of surface energy were known). In our case, deionized water and diiodomethane were chosen.

2) Prepare solid substrates. Here pure polymer films were prepared. 
3) By measuring the contact angles of the two different liquids (step 1) on the substrate (step 2), the surface energies of the substrates can be calculated according to Owens-Wendt-Kaelble and Young's equations.

4) Calculate the solubility parameter $(\delta)$.

$\delta=K \sqrt{\gamma_{S}}$

$\gamma_{\mathrm{S}}$ is the surface energy of the solid; $K$ is the proportionality constant $(K=$ $116 \times 10^{3} \mathrm{~m}^{-1 / 2}$.

5) Calculate the Flory-Huggins interaction parameter $\left(\chi_{\mathrm{DA}}\right)$.

$\chi_{D A}=\frac{V_{0}}{R_{g} T}\left(\delta_{D}-\delta_{A}\right)^{2}$

is the geometric mean of the polymer segment molar volume, $R_{\mathrm{g}}$ is the gas constant, $T$ is the absolute temperature, and $\delta_{\mathrm{D}}$ and $\delta_{\mathrm{A}}$ are the solubility parameter of the polymer donor and acceptor, respectively.

Spreading parameter $(\Delta W)$ : The spreading parameter is a useful parameter to quantify the wettability of a solution on a solid surface, which can be determined by the following procedures. ${ }^{10-13}$

1) Choose liquids 1 and 2 (whose polar and dispersive components of surface energy were known). In our case, deionized water and diiodomethane were chosen.

2) Choose two substrates 1 and 2. Here glass and silicon wafer were chosen. 
3) By measuring the contact angles of the two different liquids (step 1) on the substrates (step 2), the surface energies of the substrates can be calculated according to Owens-Wendt-Kaelble and Young's equations.

$$
\begin{aligned}
& \gamma_{L 1}\left(\cos \theta_{1,1}+1\right)=2\left(\gamma_{S 1}^{P} \gamma_{L 1}^{P}\right)^{1 / 2}+2\left(\gamma_{S 1}^{D} \gamma_{L 1}^{D}\right)^{1 / 2} \\
& \gamma_{L 2}\left(\cos \theta_{2,1}+1\right)=2\left(\gamma_{S 1}^{P} \gamma_{L 2}^{P}\right)^{1 / 2}+2\left(\gamma_{S 1}^{D} \gamma_{L 2}^{D}\right)^{1 / 2} \\
& \gamma_{S 1}=\gamma_{S 1}^{D}+\gamma_{S 1}^{P}
\end{aligned}
$$

Here $\gamma$ is the surface energy. The superscripts $\mathrm{D}$ and $\mathrm{P}$ refer to the dispersive part and the polar part respectively. The subscripts L1 and L2 refer to liquids 1 and 2, and the subscripts S1 and S2 refer to substrate 1 and 2 , respectively. $\theta_{1,1}$ is the equilibrium contact angle of liquid 1 on substrate $1 ; \theta_{2,1}$ is the equilibrium contact angle of liquid 2 on substrate 1 . Since $\gamma_{\mathrm{L} 1}{ }^{\mathrm{D}}, \gamma_{\mathrm{L} 1}{ }^{\mathrm{P}}, \gamma_{\mathrm{L} 2}{ }^{\mathrm{D}}$ and $\gamma_{\mathrm{L} 2}{ }^{\mathrm{P}}$ are known, $\gamma_{\mathrm{S} 1}{ }^{\mathrm{D}}$ and $\gamma_{\mathrm{S} 1}{ }^{\mathrm{P}}$ can be calculated by solving Equations 5 and 6, and $\gamma_{\mathrm{S} 1}$ is obtained based on Equation 7. As for substrate $2, \gamma_{\mathrm{S} 2}{ }^{\mathrm{D}}, \gamma_{\mathrm{S} 2}^{\mathrm{P}}$ and $\gamma_{\mathrm{S} 2}$ can be derived by analogy.

4) For liquid 3, a polymer solution, $\gamma_{\mathrm{L} 3}^{\mathrm{D}}$ and $\gamma_{\mathrm{L} 3}{ }^{\mathrm{P}}$ are calculated from contact angles of the solution on the two different substrates (step 2) whose polar and dispersive components are known in step 3.

$$
\begin{aligned}
& \gamma_{L 3}\left(\cos \theta_{3,1}+1\right)=2\left(\gamma_{S 1}^{P} \gamma_{L 3}^{P}\right)^{1 / 2}+2\left(\gamma_{S 1}^{D} \gamma_{L 3}^{D}\right)^{1 / 2} \\
& \gamma_{L 3}\left(\cos \theta_{3,2}+1\right)=2\left(\gamma_{S 2}^{P} \gamma_{L 3}^{P}\right)^{1 / 2}+2\left(\gamma_{S 2}^{D} \gamma_{L 3}^{D}\right)^{1 / 2} \\
& \gamma_{L 3}=\gamma_{L 3}^{D}+\gamma_{L 3}^{P}
\end{aligned}
$$


Here the subscript 3 refers to liquid 3. $\theta_{3,1}$ is the equilibrium contact angle of liquid 3 on substrate $1 . \theta_{3,2}$ is the equilibrium contact angle of liquid 3 on substrate 2 .

5) Calculate the the spreading parameter of liquid 3 (step 4) on substrate 3 (a thin layer of PEDOT:PSS on ITO in our case).

$W_{S L 3}=\gamma_{L 3}\left(\cos \theta_{3,3}+1\right)$

$W_{L L 3}=2 \gamma_{L 3}$

$\Delta W_{3}=\gamma_{L 3}\left(\cos \theta_{3,3}-1\right)$

Here $W_{\mathrm{SL}}$ represents the work of adhesion, which is required to separate a unit area of the solid-liquid interface. $W_{\mathrm{LL}}$ represents the work of cohesion, which is required to separate a unit area of liquid-liquid interface. $\Delta W$ shows the extent to which the liquid will adhere to the surface relative to itself and hence indicates the ability of a liquid drop to stick to the solid surface during solution processing. The subscript 3 refers to liquid 3 and substrate $3 . \theta_{3,3}$ is the equilibrium contact angle of liquid 3 on substrate 3. 


\section{Additional Figures and Tables}

(a)

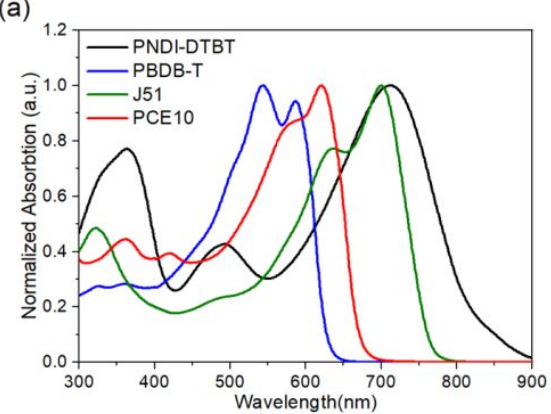

(b)

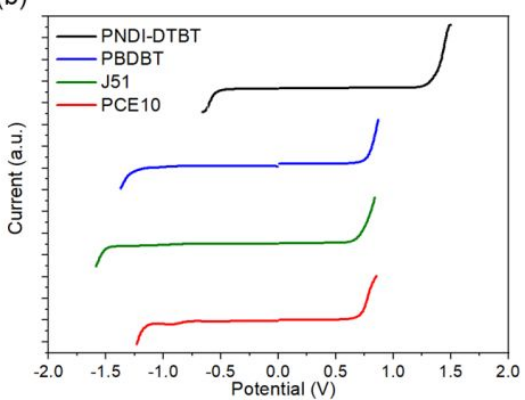

Figure S1. (a) Optical absorption spectra in solutions, and (b) square wave voltammograms of PNDI-DTBT, PBDB-T, J51, and PCE10.

Table S1. Optical properties and energy levels of PNDI-DTBT, PBDB-T, J51, and PCE10.

\begin{tabular}{lccccc}
\hline Polymer & $\begin{array}{c}\lambda_{\max }{ }^{\mathrm{a}} \\
{[\mathrm{nm}]}\end{array}$ & $\begin{array}{c}\lambda_{\text {onset }^{\mathrm{a}}}[\mathrm{nm}]\end{array}$ & $\begin{array}{c}E_{\mathrm{g}} \mathrm{optb} \\
{[\mathrm{eV}]}\end{array}$ & $\begin{array}{c}\text { HOMO }^{\mathrm{c}} \\
{[\mathrm{eV}]}\end{array}$ & $\begin{array}{c}\text { LUMOd) } \\
{[\mathrm{eV}]}\end{array}$ \\
\hline PNDI-DTBT & 366,693 & 833 & 1.49 & -5.77 & -3.96 \\
PBDB-T & 582,624 & 676 & 1.83 & -5.27 & -3.22 \\
J51 & 549,598 & 645 & 1.92 & -5.17 & -3.00 \\
PCE10 & 638,697 & 760 & 1.63 & -5.22 & -3.34 \\
\hline
\end{tabular}

a) In films. ${ }^{\text {b) }} E_{\mathrm{g}}{ }^{\text {opt }}=1240 / \lambda_{\text {onset. }}{ }^{\text {c) }}$ HOMO energy levels were estimated from the oxidation onset potential. d)LUMO energy levels were estimated from the reduction onset potential. 
Table S2. Device performance of the all-PSCs based on PBDB-T:PNDI-DTBT ${ }^{\text {a) }}$ with different fabrication conditions under AM1.5G illumination (100 $\left.\mathrm{mW} \mathrm{cm}^{-2}\right)$.

\begin{tabular}{|c|c|c|c|c|c|c|}
\hline $\begin{array}{l}\text { D:A ratio } \\
{[\mathrm{w} / \mathrm{w}]}\end{array}$ & $\begin{array}{c}\text { Solvent } \\
{[\mathrm{v} / \mathrm{v}]}\end{array}$ & Annealing & $\begin{array}{l}V_{\mathrm{oc}} \\
{[\mathrm{V}]}\end{array}$ & $\begin{array}{c}J_{\mathrm{sc}} \\
{\left[\mathrm{mA} \mathrm{cm}^{-2}\right]}\end{array}$ & FF & $\begin{array}{l}\text { PCE } \\
{[\%]}\end{array}$ \\
\hline $1: 2$ & \multirow{5}{*}{ СВ } & \multirow{5}{*}{$\mathrm{w} / \mathrm{o}$} & 0.86 & 10.2 & 0.43 & 3.8 \\
\hline $1: 1.5$ & & & 0.87 & 12.1 & 0.47 & 4.9 \\
\hline $1: 1$ & & & 0.86 & 12.6 & 0.51 & 5.5 \\
\hline $1.5: 1$ & & & 0.87 & 12.3 & 0.51 & 5.4 \\
\hline $1: 2$ & & & 0.86 & 10.2 & 0.43 & 3.8 \\
\hline \multirow{3}{*}{$1: 1$} & СB & \multirow{3}{*}{$\mathrm{w} / \mathrm{o}$} & 0.86 & 12.6 & 0.51 & 5.5 \\
\hline & $\mathrm{BB}^{\mathrm{b})}$ & & 0.89 & 13.2 & 0.54 & 6.3 \\
\hline & $o$-DCB & & 0.84 & 6.0 & 0.47 & 2.4 \\
\hline \multirow{4}{*}{$1: 1$} & \multirow{4}{*}{$\mathrm{BB}$} & $\mathrm{w} / \mathrm{o}$ & 0.89 & 13.2 & 0.54 & 6.3 \\
\hline & & $60^{\circ} \mathrm{C}, 10 \mathrm{~min}$ & 0.88 & 12.4 & 0.54 & 5.9 \\
\hline & & $90^{\circ} \mathrm{C}, 10 \mathrm{~min}$ & 0.87 & 12.2 & 0.54 & 5.7 \\
\hline & & $120^{\circ} \mathrm{C}, 10 \mathrm{~min}$ & 0.81 & 13.7 & 0.41 & 4.5 \\
\hline \multirow{4}{*}{$1: 1$} & $\mathrm{BB} / 0.5 \% \mathrm{DIO}^{\mathrm{c}}$ & \multirow{4}{*}{$\mathrm{w} / \mathrm{o}$} & 0.89 & 10.5 & 0.57 & 5.4 \\
\hline & $\mathrm{BB} / 0.5 \% \mathrm{DPE}^{\mathrm{d})}$ & & 0.88 & 11.9 & 0.58 & 6.1 \\
\hline & $\mathrm{BB} / 0.5 \% \mathrm{ODT}^{\mathrm{e}}$ & & 0.86 & 9.3 & 0.47 & 3.8 \\
\hline & $\mathrm{BB} / 0.5 \% \mathrm{CN}^{\mathrm{f}}$ & & 0.89 & 12.3 & 0.54 & 5.9 \\
\hline \multirow{5}{*}{$1: 1$} & \multirow{5}{*}{ СВ } & $\mathrm{w} / \mathrm{o}$ & 0.86 & 12.1 & 0.51 & 5.3 \\
\hline & & $60^{\circ} \mathrm{C}, 10 \mathrm{~min}$ & 0.89 & 12.1 & 0.53 & 5.7 \\
\hline & & $90^{\circ} \mathrm{C}, 10 \mathrm{~min}$ & 0.89 & 12.1 & 0.54 & 5.8 \\
\hline & & $120^{\circ} \mathrm{C}, 10 \mathrm{~min}$ & 0.88 & 12.6 & 0.54 & 6.0 \\
\hline & & $150^{\circ} \mathrm{C}, 10 \mathrm{~min}$ & 0.88 & 11.9 & 0.54 & 5.6 \\
\hline \multirow{5}{*}{$1: 1$} & $\mathrm{CB}$ & \multirow{5}{*}{$\mathrm{w} / \mathrm{o}$} & 0.86 & 12.1 & 0.51 & 5.3 \\
\hline & CB $/ 1 \%$ DIO & & 0.88 & 12.5 & 0.52 & 5.8 \\
\hline & $\mathrm{CB} / 1 \%$ ODT & & 0.87 & 13.2 & 0.51 & 5.9 \\
\hline & $\mathrm{CB} / 1 \% \mathrm{DPE}$ & & 0.89 & 12.6 & 0.51 & 5.7 \\
\hline & $\mathrm{CB} / 1 \% \mathrm{CN}$ & & 0.69 & 6.4 & 0.36 & 1.6 \\
\hline \multirow{5}{*}{$1: 1$} & $\mathrm{CB}$ & \multirow{5}{*}{$\mathrm{w} / \mathrm{o}$} & 0.86 & 12.1 & 0.51 & 5.3 \\
\hline & $\mathrm{CB} / 0.25 \%$ ODT & & 0.85 & 13.3 & 0.48 & 5.4 \\
\hline & $\mathrm{CB} / 0.5 \%$ ODT & & 0.88 & 12.9 & 0.49 & 5.5 \\
\hline & $\mathrm{CB} / 1 \% \mathrm{ODT}$ & & 0.87 & 13.2 & 0.51 & 5.9 \\
\hline & $\mathrm{CB} / 2 \%$ ODT & & 0.89 & 12.2 & 0.51 & 5.5 \\
\hline 1:1 & CB/1\% ODT & $120^{\circ} \mathrm{C}, 10 \mathrm{~min}$ & 0.90 & 14.5 & 0.64 & 8.5 \\
\hline
\end{tabular}

a)PNDI-DTBT has a number-average molecular weight and a molar-mass dispersity of $97.2 \mathrm{kDa}$ and 1.9 , respectively. ${ }^{\text {b) }} \mathrm{BB}$ is bromobenzene. ${ }^{\mathrm{c}} \mathrm{DIO}$ is $1,8-$ Diiodooctane. d)DPE is diphenyl ether. e) ODT is $1,8-$-Octanedithiol. ${ }^{\text {f) }} \mathrm{CN}$ is chloronaphthalene. 
Table S3. The device performance of the PBDB-T:PNDI-DTBT all-PSCs with different molecular weight of PNDI-DTBT under AM1.5G illumination $(100 \mathrm{~mW}$ $\left.\mathrm{cm}^{-2}\right)^{\text {a). }}$.

\begin{tabular}{cccccc}
\hline $\begin{array}{c}M_{\mathrm{n}} \\
{[\mathrm{kDa}]}\end{array}$ & $\Xi_{\mathrm{M}}$ & $\begin{array}{c}V_{\mathrm{oc}} \\
{[\mathrm{V}]}\end{array}$ & $\begin{array}{c}J_{\mathrm{sc}} \\
{\left[\mathrm{mA} \mathrm{cm}^{-2}\right]}\end{array}$ & FF & $\begin{array}{c}\text { PCE } \\
{[\%]}\end{array}$ \\
\hline 103.9 & 1.58 & 0.89 & 13.7 & 0.64 & 7.8 \\
97.2 & 1.85 & 0.90 & 14.5 & 0.64 & 8.5 \\
87.9 & 1.87 & 0.88 & 13.9 & 0.64 & 7.9 \\
\hline
\end{tabular}

a)All-PSCs fabrication parameters: a D:A ratio of 1:1, a solvent mixture of chlorobenzene with $1 \%$ ODT in volume, thermally annealed at $120{ }^{\circ} \mathrm{C}$ for 10 minutes.

Table S4. Device performance of the all-PSCs based on J51:PNDI-DTBTa) with different fabrication conditions under AM1.5G illumination $\left(100 \mathrm{~mW} \mathrm{~cm}^{-2}\right)$.

\begin{tabular}{|c|c|c|c|c|c|c|}
\hline $\begin{array}{l}\text { D:A ratio } \\
{[w / w]}\end{array}$ & $\begin{array}{c}\text { Solvent } \\
{[\mathrm{v} / \mathrm{v}]}\end{array}$ & Annealing & $\begin{array}{l}V_{\mathrm{oc}} \\
{[\mathrm{V}]}\end{array}$ & $\begin{array}{c}J_{\mathrm{sc}} \\
{\left[\mathrm{mA} \mathrm{cm}^{-2}\right]}\end{array}$ & FF & $\begin{array}{l}\text { PCE } \\
{[\%]}\end{array}$ \\
\hline $2.5: 1$ & & & 0.84 & 7.1 & 0.45 & 2.7 \\
\hline $2: 1$ & & & 0.85 & 9.0 & 0.46 & 3.5 \\
\hline $1: 1.5$ & $o$-DCB & $\mathrm{w} / \mathrm{o}$ & 0.85 & 7.7 & 0.46 & 3.0 \\
\hline $1: 1$ & & & 0.84 & 7.1 & 0.46 & 2.7 \\
\hline $1: 2$ & & & 0.84 & 6.4 & 0.45 & 2.4 \\
\hline \multirow{4}{*}{$2: 1$} & \multirow{4}{*}{$o$-DCB } & $80^{\circ} \mathrm{C}, 10 \mathrm{~min}$ & 0.84 & 10.6 & 0.49 & 4.4 \\
\hline & & $100^{\circ} \mathrm{C}, 10 \mathrm{~min}$ & 0.85 & 12.2 & 0.49 & 5.3 \\
\hline & & $120^{\circ} \mathrm{C}, 10 \mathrm{~min}$ & 0.84 & 11.0 & 0.53 & 4.9 \\
\hline & & $140^{\circ} \mathrm{C}, 10 \mathrm{~min}$ & 0.84 & 10.9 & 0.51 & 4.7 \\
\hline \multirow{3}{*}{$2: 1$} & $\mathrm{CB}$ & \multirow{3}{*}{$w / o$} & 0.84 & 8.4 & 0.41 & 2.9 \\
\hline & $o-\mathrm{DCB}$ & & 0.85 & 8.9 & 0.46 & 3.5 \\
\hline & BB & & 0.84 & 11.4 & 0.46 & 4.4 \\
\hline \multirow{5}{*}{$2: 1$} & $\mathrm{BB}$ & \multirow{5}{*}{$w / o$} & 0.84 & 11.4 & 0.46 & 4.4 \\
\hline & $\mathrm{BB} / 1 \% \mathrm{DIO}$ & & 0.83 & 12.2 & 0.50 & 5.1 \\
\hline & $\mathrm{BB} / 1 \% \mathrm{DPE}$ & & 0.84 & 12.8 & 0.57 & 6.1 \\
\hline & $\mathrm{BB} / 1 \% \mathrm{ODT}$ & & 0.84 & 8.0 & 0.45 & 3.0 \\
\hline & $\mathrm{BB} / 1 \% \mathrm{CN}$ & & 0.85 & 6.3 & 0.52 & 2.8 \\
\hline \multirow{6}{*}{$2: 1$} & BB & \multirow{6}{*}{$w / o$} & 0.84 & 11.4 & 0.46 & 4.4 \\
\hline & $\mathrm{BB} / 0.5 \% \mathrm{DPE}$ & & 0.84 & 12.2 & 0.59 & 6.0 \\
\hline & $\mathrm{BB} / 0.75 \% \mathrm{DPE}$ & & 0.84 & 12.4 & 0.59 & 6.2 \\
\hline & $\mathrm{BB} / 1 \% \mathrm{DPE}$ & & 0.84 & 12.8 & 0.57 & 6.1 \\
\hline & $\mathrm{BB} / 1.25 \% \mathrm{DPE}$ & & 0.84 & 12.8 & 0.56 & 6.1 \\
\hline & $\mathrm{BB} / 1.5 \% \mathrm{DPE}$ & & 0.84 & 12.8 & 0.55 & 5.9 \\
\hline $2: 1$ & BB/0.75\%DPE & $100^{\circ} \mathrm{C}, 10 \mathrm{~min}$ & 0.85 & 12.9 & 0.60 & 6.5 \\
\hline
\end{tabular}

a)PNDI-DTBT has a number-average molecular weight and a molar-mass dispersity of $97.2 \mathrm{kDa}$ and 1.9 , respectively. 
Table S5. Device performance of the all-PSCs based on PCE10:PNDI-DTBT ${ }^{\mathrm{a})}$ with different fabrication conditions under AM1.5G illumination $\left(100 \mathrm{~mW} \mathrm{~cm}^{-2}\right)$.

\begin{tabular}{|c|c|c|c|c|c|c|}
\hline $\begin{array}{l}\text { D:A ratio } \\
{[\mathrm{w} / \mathrm{w}]}\end{array}$ & $\begin{array}{c}\text { Solvent } \\
{[\mathrm{v} / \mathrm{v}]}\end{array}$ & Annealing & $\begin{array}{l}V_{\mathrm{oc}} \\
{[\mathrm{V}]}\end{array}$ & $\begin{array}{c}J_{\mathrm{sc}} \\
{\left[\mathrm{mA} \mathrm{cm}^{-2}\right]}\end{array}$ & $\mathrm{FF}$ & $\begin{array}{l}\text { PCE } \\
{[\%]}\end{array}$ \\
\hline $2: 1$ & \multirow{5}{*}{ CB } & \multirow{5}{*}{$\mathrm{w} / \mathrm{o}$} & 0.81 & 13.2 & 0.47 & 5.0 \\
\hline $1.5: 1$ & & & 0.81 & 12.6 & 0.49 & 5.0 \\
\hline $1: 1$ & & & 0.84 & 13.3 & 0.54 & 5.8 \\
\hline $1: 1.5$ & & & 0.81 & 12.4 & 0.47 & 4.7 \\
\hline $1: 2$ & & & 0.82 & 10.9 & 0.44 & 3.9 \\
\hline \multirow{3}{*}{$1: 1$} & $\mathrm{CB}$ & \multirow{3}{*}{$\mathrm{w} / \mathrm{o}$} & 0.84 & 13.3 & 0.53 & 5.8 \\
\hline & $o$-DCB & & 0.81 & 11.3 & 0.50 & 4.6 \\
\hline & BB & & 0.81 & 9.1 & 0.54 & 4.0 \\
\hline \multirow{3}{*}{$1: 1$} & \multirow{3}{*}{ CB } & $\mathrm{w} / \mathrm{o}$ & 0.84 & 13.3 & 0.53 & 5.8 \\
\hline & & $90^{\circ} \mathrm{C}, 10 \mathrm{~min}$ & 0.82 & 12.4 & 0.50 & 5.1 \\
\hline & & $120^{\circ} \mathrm{C}, 10 \mathrm{~min}$ & 0.82 & 12.3 & 0.49 & 5.0 \\
\hline \multirow{4}{*}{$1: 1$} & CB & \multirow{4}{*}{$w / o$} & 0.84 & 13.3 & 0.53 & 5.8 \\
\hline & CB/1\%DIO & & 0.83 & 10.7 & 0.52 & 4.6 \\
\hline & $\mathrm{CB} / 1 \% \mathrm{DPE}$ & & 0.81 & 11.1 & 0.50 & 4.5 \\
\hline & CB/1\%ODT & & 0.82 & 10.9 & 0.55 & 5.0 \\
\hline
\end{tabular}

a)PNDI-DTBT has a number-average molecular weight and a molar-mass dispersity of $97.2 \mathrm{kDa}$ and 1.9 , respectively.

Table S6. The device performance of the all-PSCs based on PBDB-T:PNDI-DTBT, J51:PNDI-DTBT, and PCE10:PNDI-DTBT under the same fabrication conditions.

\begin{tabular}{|c|c|c|c|c|c|c|c|}
\hline $\begin{array}{l}\text { Active } \\
\text { layer }\end{array}$ & $\begin{array}{c}\text { D:A ratio } \\
{[\mathrm{w} / \mathrm{w}]}\end{array}$ & $\begin{array}{c}\text { Solvent } \\
{[\mathrm{v} / \mathrm{v}]}\end{array}$ & Annealing & $\begin{array}{l}V_{\text {oc }} \\
{[\mathrm{V}]}\end{array}$ & $\begin{array}{c}J_{\mathrm{sc}} \\
{\left[\mathrm{mA} \mathrm{cm}^{-2}\right]}\end{array}$ & $\mathrm{FF}$ & $\begin{array}{l}\text { PCE } \\
{[\%]}\end{array}$ \\
\hline \multirow{3}{*}{$\begin{array}{l}\text { PBDB-T: } \\
A^{\text {a) }}\end{array}$} & \multirow{3}{*}{$1: 1$} & $\mathrm{CB}$ & $\mathrm{w} / \mathrm{o}$ & 0.86 & 12.1 & 0.51 & 5.3 \\
\hline & & $\mathrm{CB} / 1 \% \mathrm{ODT}$ & $\mathrm{w} / \mathrm{o}$ & 0.87 & 13.2 & 0.51 & 5.9 \\
\hline & & CB/1\%ODT & $120^{\circ} \mathrm{C}, 10 \mathrm{~min}$ & 0.90 & 14.5 & 0.64 & 8.5 \\
\hline \multirow{3}{*}{ J51:A } & \multirow{3}{*}{$1: 1$} & CB & $\mathrm{w} / \mathrm{o}$ & 0.81 & 13.1 & 0.52 & 5.5 \\
\hline & & CB/1\%ODT & $\mathrm{w} / \mathrm{o}$ & 0.83 & 12.8 & 0.55 & 5.9 \\
\hline & & CB/1\%ODT & $120^{\circ} \mathrm{C}, 10 \mathrm{~min}$ & 0.82 & 13.2 & 0.57 & 6.2 \\
\hline \multirow{3}{*}{ PCE10:A } & \multirow{3}{*}{$1: 1$} & СB & $\mathrm{w} / \mathrm{o}$ & 0.84 & 13.3 & 0.53 & 5.8 \\
\hline & & CB/1\%ODT & $\mathrm{w} / \mathrm{o}$ & 0.82 & 10.9 & 0.55 & 5.0 \\
\hline & & CB/1\%ODT & $120^{\circ} \mathrm{C}, 10 \mathrm{~min}$ & 0.83 & 10.7 & 0.52 & 4.6 \\
\hline
\end{tabular}

a)The A represents the polymer acceptor PNDI-DTBT. 
Table S7. Summary of high-performance (PCE $\geq 8.0 \%$ ) all-PSCs with polymer acceptors beyond N2200-based polymers and the polymer acceptors derived from fused-ring non-fullerene small molecules.

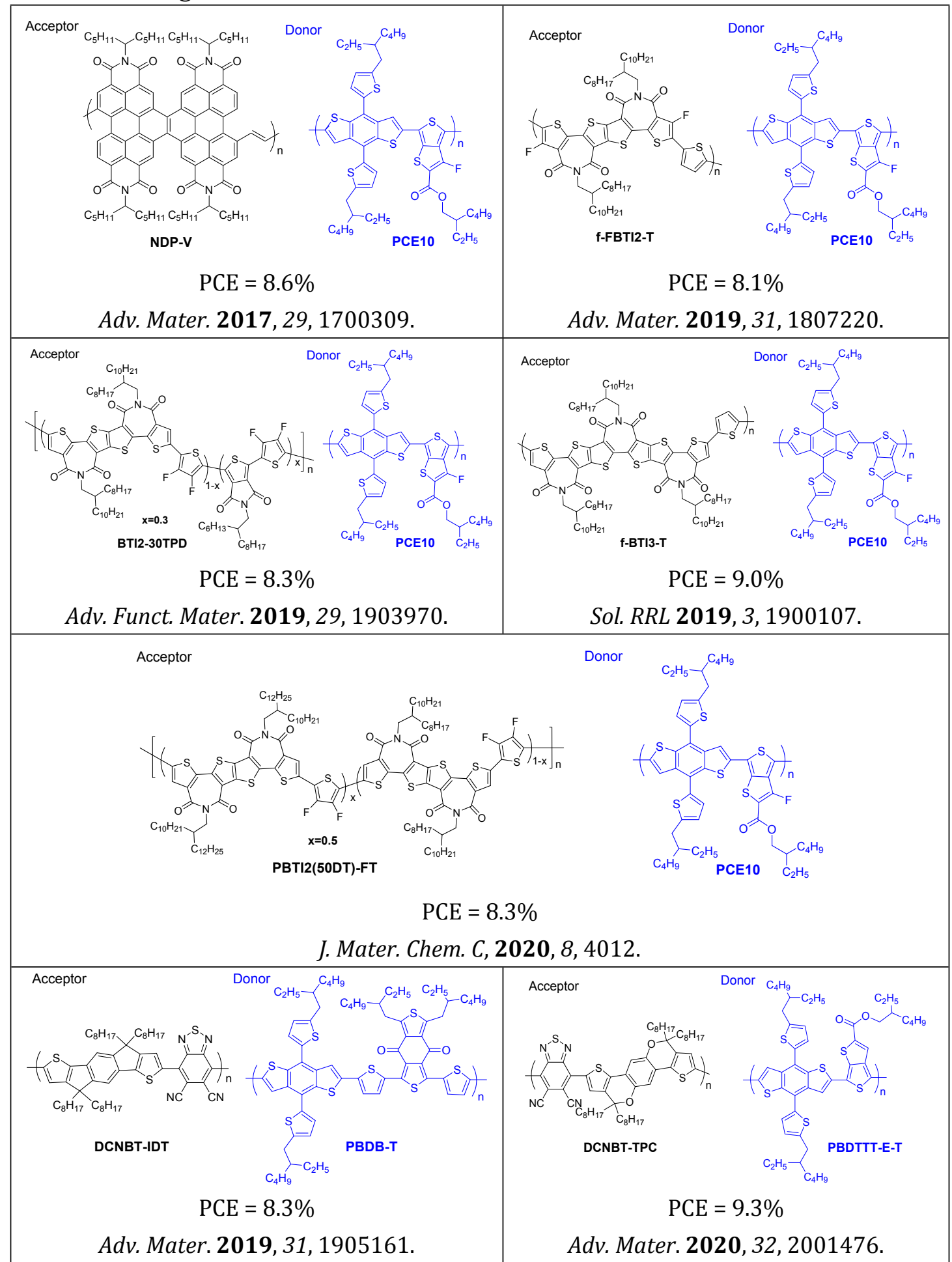



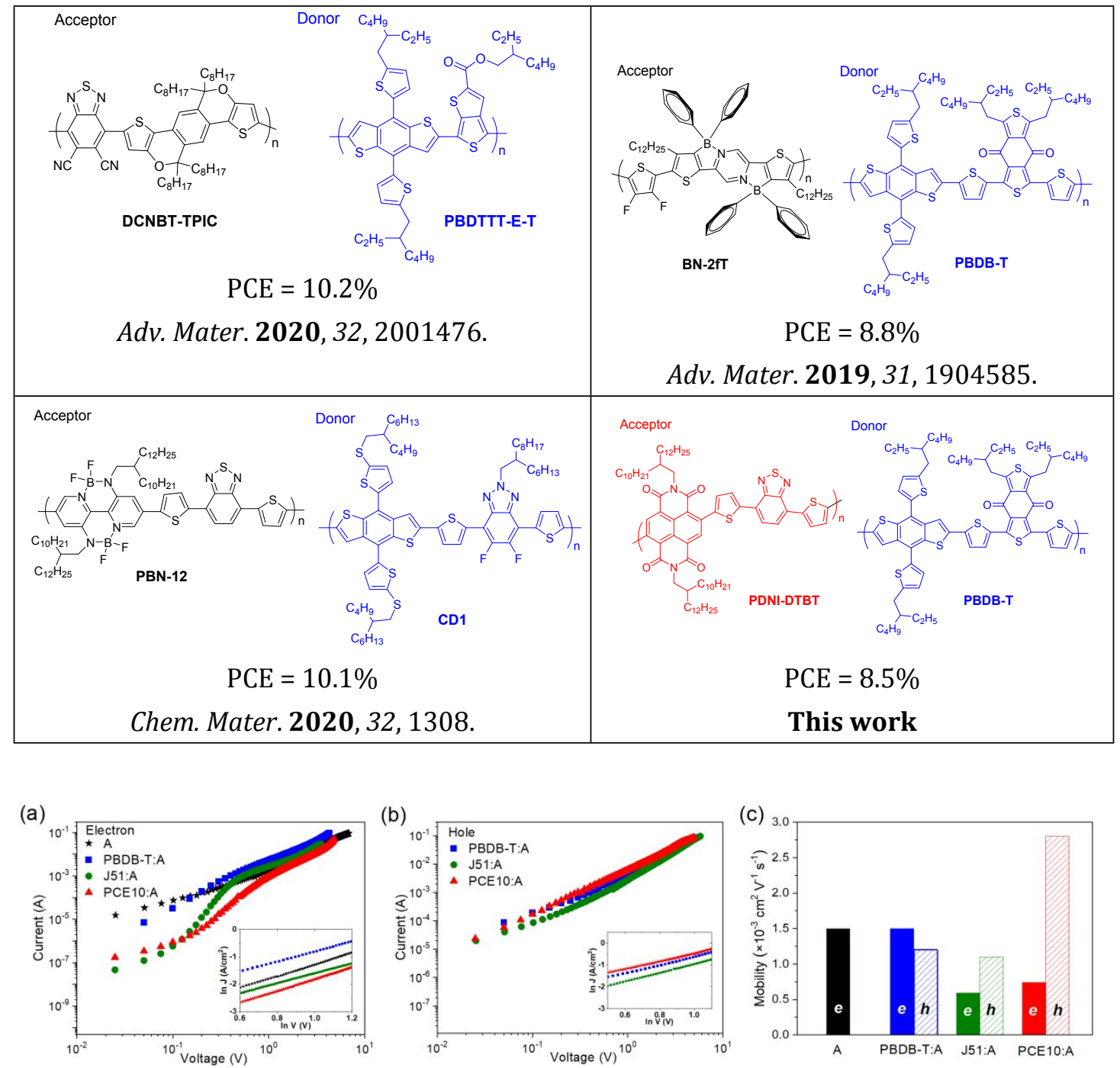

Figure S2. Current density versus voltage characteristics of (a) electron-only devices with a structure of ITO/ZnO/conducting layer/PFN-Br/Ag, and (b) holeonly devices with a structure of ITO/PEDOT:PSS/conducting layer $/ \mathrm{MoO}_{3} / \mathrm{Ag}$. The insets show the fitting region. (c) Hole and electron mobility acquired from singlecarrier devices based on the three donor:acceptor blends. A represents the polymer acceptor PNDI-DTBT, $e$ represents electron mobility, and $h$ represents hole mobility.

Table S8. Charge carrier mobilities of PNDI-DTBT and various donor:acceptor blends in single-carrier devices.

\begin{tabular}{lccc}
\hline Active layer & $\begin{array}{c}\mu_{\mathrm{e}} \\
{\left[\mathrm{cm}^{2} \mathrm{~V}^{-1} \mathrm{~s}^{-1}\right]}\end{array}$ & $\begin{array}{c}\mu_{\mathrm{h}} \\
{\left[\mathrm{cm}^{2} \mathrm{~V}^{-1} \mathrm{~s}^{-1}\right]}\end{array}$ & $\mu_{\mathrm{e}} / \mu_{\mathrm{h}}$ \\
\hline PNDI-DTBT & $1.5 \times 10^{-3}$ & - & - \\
PBDB-T:PNDI-DTBT & $1.5 \times 10^{-3}$ & $1.2 \times 10^{-3}$ & 1.25 \\
J51:PNDI-DTBT & $5.9 \times 10^{-4}$ & $1.1 \times 10^{-3}$ & 0.53 \\
PCE10:PNDI-DTBT & $7.4 \times 10^{-4}$ & $2.8 \times 10^{-3}$ & 0.26 \\
\hline
\end{tabular}



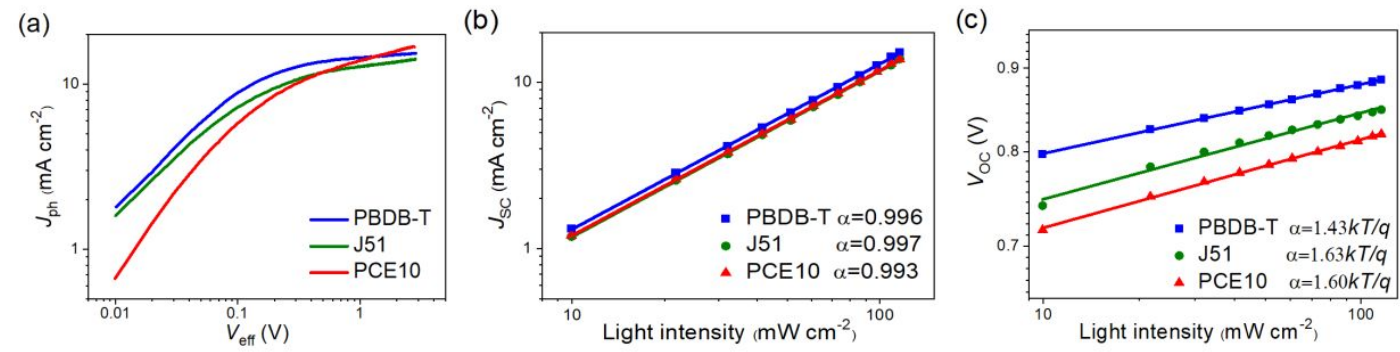

Figure S3. (a) Photocurrent density $\left(U_{\mathrm{ph}}\right)$ as a function of effective voltage $\left(V_{\text {eff }}\right)$, (b) light intensity dependence of $J_{\mathrm{sc}}$ and (c) light intensity dependence of $V_{\mathrm{oc}}$ of the all-PSCs based on PNDI-DTBT and various polymer donors.
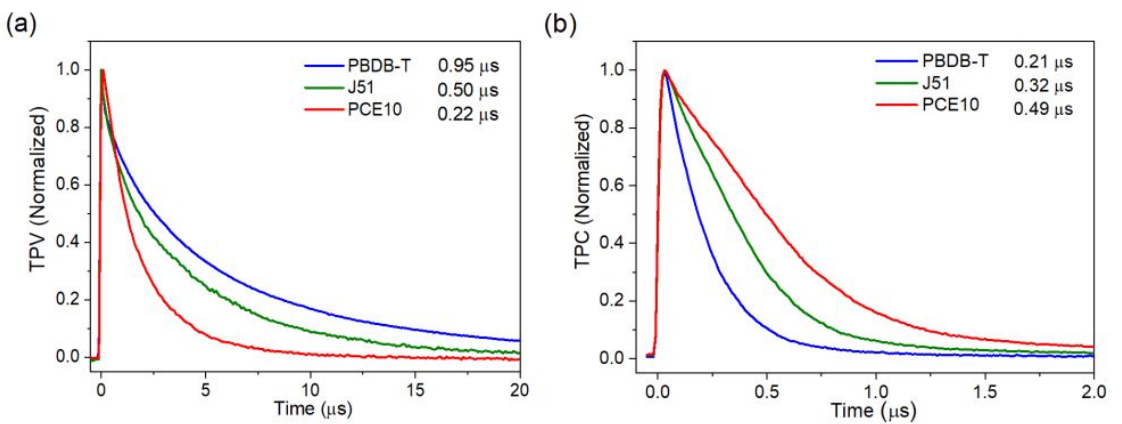

Figure S4. (a) TPV and (b) TPC curves of the all-PSCs based on PNDI-DTBT and various polymer donors.
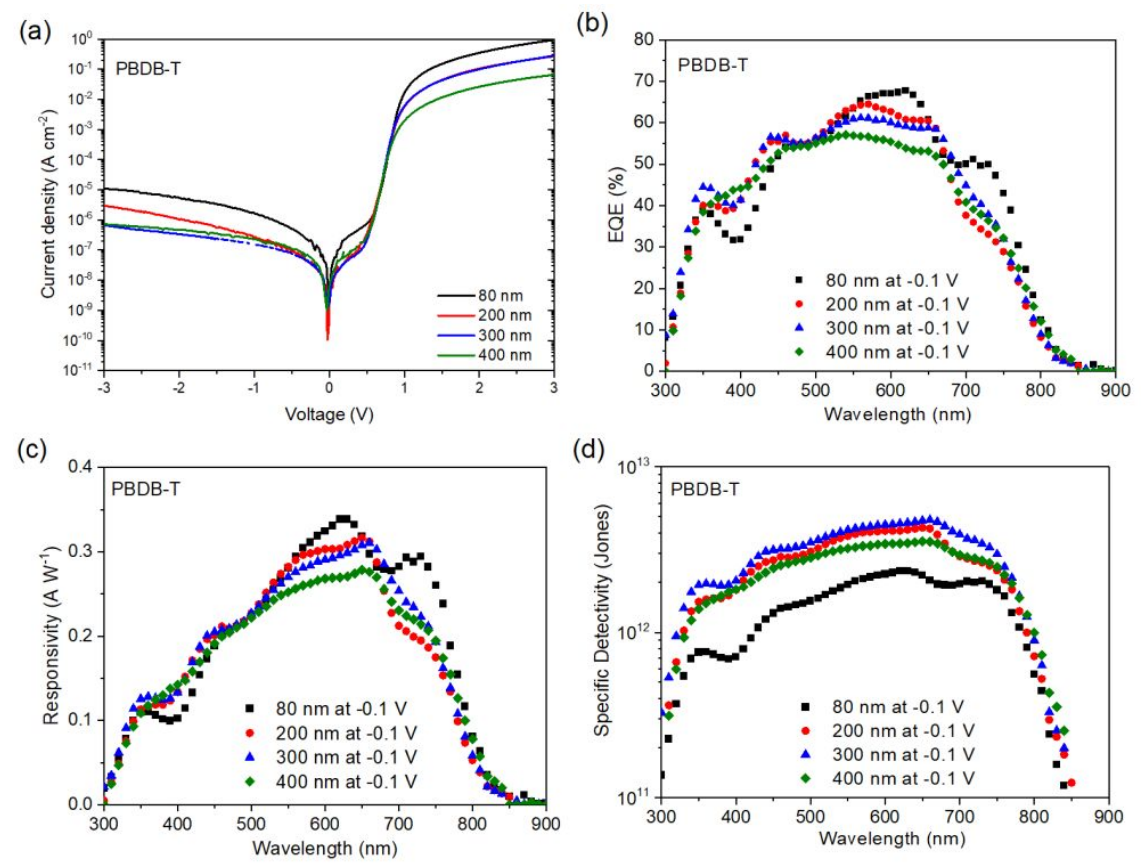

Figure S5. Device performance of all-PPDs based on PBDB-T:PNDI-DTBT with different active layer thickness. (a) Current density-voltage characteristics in dark. (b) EQE spectra, (c) the responsivity of the corresponding and (d) the specific detectivity of the corresponding devices at $-0.1 \mathrm{~V}$ bias. 
Table S9. Device parameters of all-PPDs based on PBDB-T:PNDI-DTBT with different active layer thickness.

\begin{tabular}{lcccc}
\hline $\begin{array}{l}\text { Thickness } \\
{[\mathrm{nm}]}\end{array}$ & $\begin{array}{c}\text { Rectification } \\
\text { ratio at } \pm 2 \mathrm{~V}\end{array}$ & $\begin{array}{c}J_{\mathrm{d}} \text { at }-0.1 \mathrm{~V} \\
{\left[\mathrm{~A} \mathrm{~cm}^{-2}\right]}\end{array}$ & $\begin{array}{c}R_{\max } \\
{\left[\mathrm{A} \mathrm{W}^{-1}\right]}\end{array}$ & $\begin{array}{c}D^{*}{ }_{\max } \\
{[J o n e s]}\end{array}$ \\
\hline 80 & $6.4 \times 10^{4}$ & $6.53 \times 10^{-8}$ & $0.34 @ 620 \mathrm{~nm}$ & $2.35 \times 10^{12} @ 620 \mathrm{~nm}$ \\
200 & $1.0 \times 10^{5}$ & $1.70 \times 10^{-8}$ & $0.32 @ 650 \mathrm{~nm}$ & $4.34 \times 10^{12} @ 650 \mathrm{~nm}$ \\
300 & $3.0 \times 10^{5}$ & $1.32 \times 10^{-8}$ & $0.31 @ 660 \mathrm{~nm}$ & $4.77 \times 10^{12} @ 660 \mathrm{~nm}$ \\
400 & $5.7 \times 10^{4}$ & $1.68 \times 10^{-8}$ & $0.28 @ 650 \mathrm{~nm}$ & $3.82 \times 10^{12} @ 650 \mathrm{~nm}$ \\
\hline
\end{tabular}
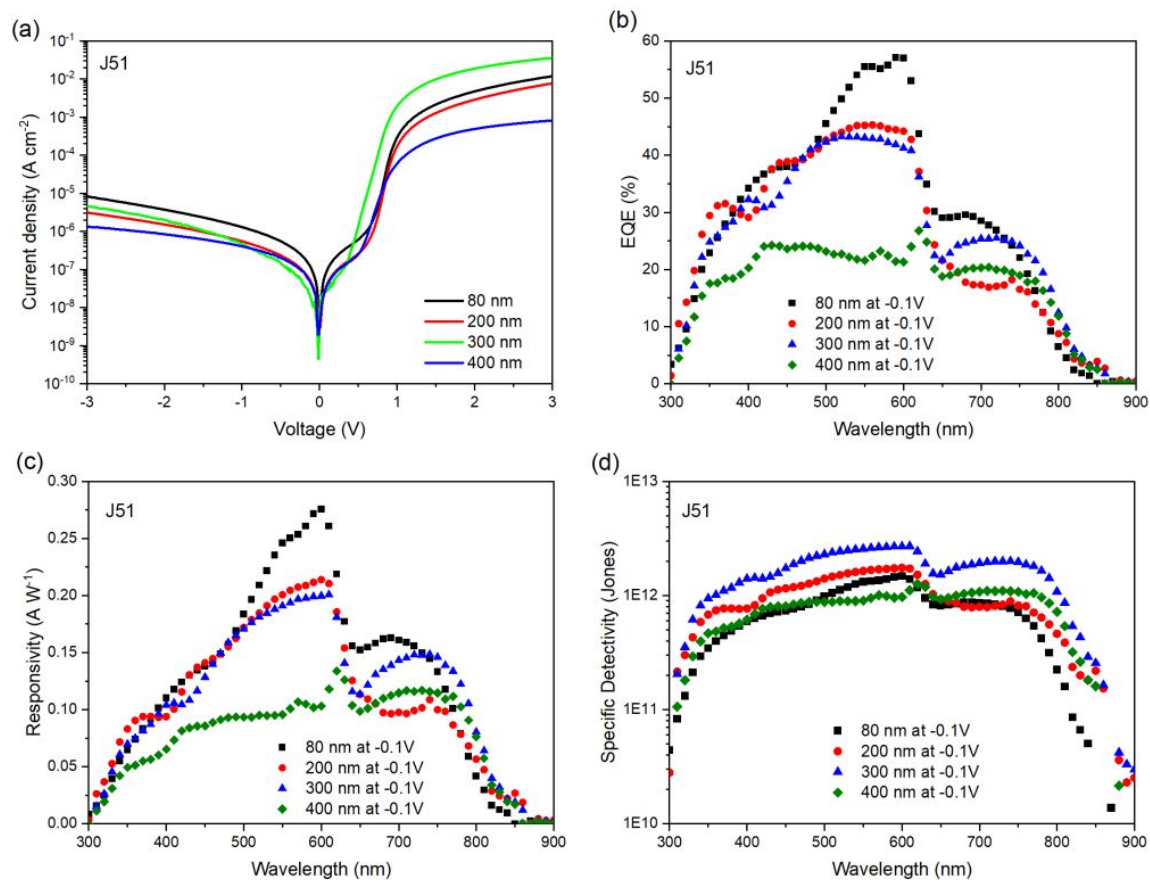

Figure S6. Device performance of all-PPDs based on J51:PNDI-DTBT with different active layer thickness. (a) Current density-voltage characteristics in dark. (b) EQE spectra, (c) the responsivity of the corresponding and (d) the specific detectivity of the corresponding devices at $-0.1 \mathrm{~V}$ bias.

Table S10. Device parameters of all-PPDs based on J51:PNDI-DTBT with different active layer thickness.

\begin{tabular}{lcccc}
\hline $\begin{array}{l}\text { Thickness } \\
{[\mathrm{nm}]}\end{array}$ & $\begin{array}{c}\text { Rectification } \\
\text { ratio at } \pm 2 \mathrm{~V}\end{array}$ & $\begin{array}{c}J_{\mathrm{d}} \text { at }-0.1 \mathrm{~V} \\
{\left[\mathrm{~A} \mathrm{~cm}^{-2}\right]}\end{array}$ & $\begin{array}{c}R_{\max } \\
{\left[\mathrm{A} \mathrm{W}^{-1}\right]}\end{array}$ & $\begin{array}{c}D_{\text {max }}^{*} \\
{[\mathrm{~J} \text { mes] }]}\end{array}$ \\
\hline 80 & $1.3 \times 10^{3}$ & $1.09 \times 10^{-7}$ & $0.28 @ 600 \mathrm{~nm}$ & $1.48 \times 10^{12} @ 600 \mathrm{~nm}$ \\
200 & $1.9 \times 10^{3}$ & $4.67 \times 10^{-8}$ & $0.21 @ 600 \mathrm{~nm}$ & $1.75 \times 10^{12} @ 600 \mathrm{~nm}$ \\
300 & $9.4 \times 10^{3}$ & $1.72 \times 10^{-8}$ & $0.20 @ 610 \mathrm{~nm}$ & $2.70 \times 10^{12} @ 610 \mathrm{~nm}$ \\
400 & $5.8 \times 10^{2}$ & $3.52 \times 10^{-8}$ & $0.13 @ 620 \mathrm{~nm}$ & $1.26 \times 10^{12} @ 620 \mathrm{~nm}$ \\
\hline
\end{tabular}



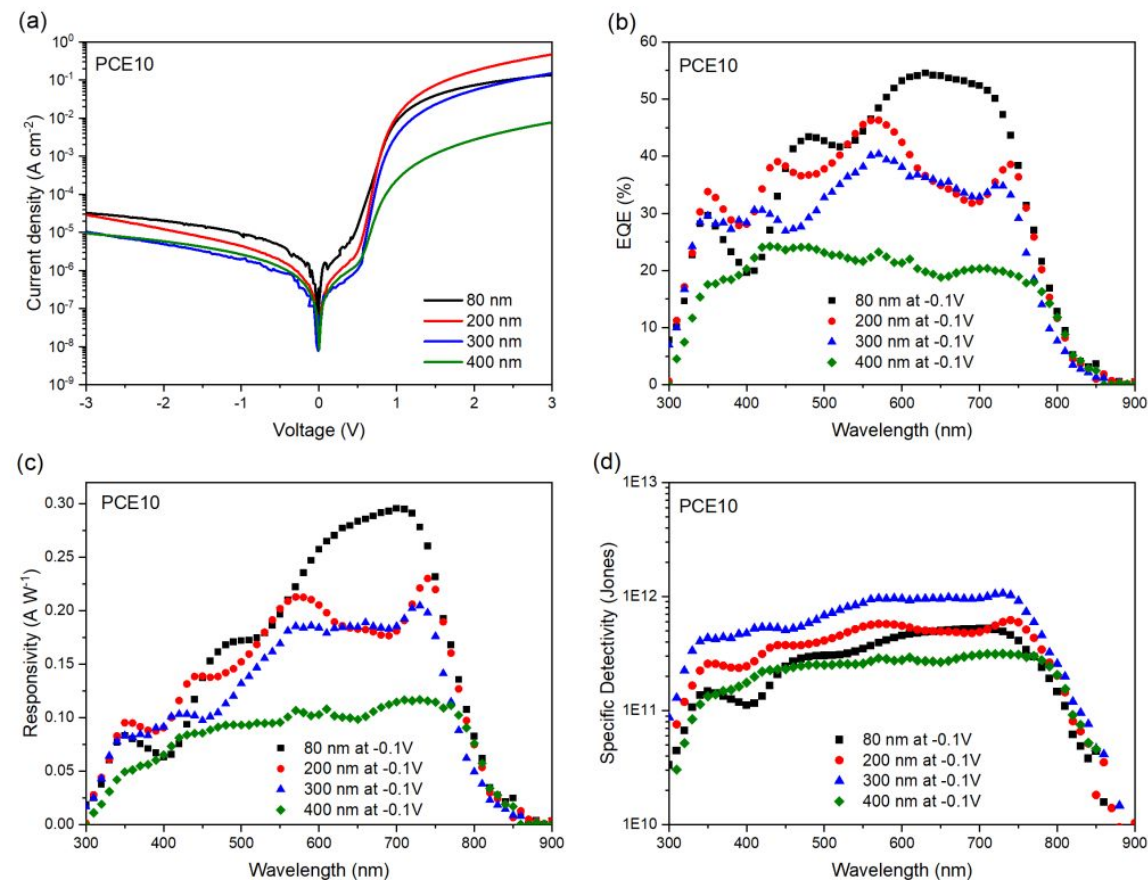

Figure S7 . Device performance of all-PPDs based on PCE10:PNDI-DTBT with different active layer thickness. (a) Current density-voltage characteristics in dark. (b) EQE spectra, (c) the responsivity of the corresponding and (d) the specific detectivity of the corresponding devices at $-0.1 \mathrm{~V}$ bias.

Table S11. Device parameters of all-PPDs based on PCE10:PNDI-DTBT with different active layer thickness.

\begin{tabular}{lcccc}
\hline $\begin{array}{l}\text { Thickness } \\
{[\mathrm{nm}]}\end{array}$ & $\begin{array}{c}\text { Rectification } \\
\text { ratio at } \pm 2 \mathrm{~V}\end{array}$ & $\begin{array}{c}J_{\mathrm{d}} \text { at }-0.1 \mathrm{~V} \\
{\left[\mathrm{~A} \mathrm{~cm}^{-2}\right]}\end{array}$ & $\begin{array}{c}R_{\max } \\
{\left[\mathrm{A} \mathrm{W}^{-1}\right]}\end{array}$ & $\begin{array}{c}D_{\max }^{*} \\
\text { [Jones] }\end{array}$ \\
\hline 80 & $3.6 \times 10^{3}$ & $9.98 \times 10^{-7}$ & $0.30 @ 700 \mathrm{~nm}$ & $5.23 \times 10^{11} @ 700 \mathrm{~nm}$ \\
200 & $1.5 \times 10^{4}$ & $4.28 \times 10^{-7}$ & $0.23 @ 740 \mathrm{~nm}$ & $6.22 \times 10^{11} @ 740 \mathrm{~nm}$ \\
300 & $1.1 \times 10^{4}$ & $1.16 \times 10^{-7}$ & $0.20 @ 730 \mathrm{~nm}$ & $1.04 \times 10^{12} @ 730 \mathrm{~nm}$ \\
400 & $4.7 \times 10^{2}$ & $2.49 \times 10^{-7}$ & $0.12 @ 710 \mathrm{~nm}$ & $3.15 \times 10^{11} @ 710 \mathrm{~nm}$ \\
\hline
\end{tabular}


Table S12. Characteristic length scale of the neat polymers and donor:acceptor blends.

\begin{tabular}{|c|c|c|c|c|c|c|}
\hline \multirow[b]{2}{*}{ Film } & \multicolumn{3}{|c|}{$(010) \pi-\pi$ stacking } & \multicolumn{3}{|c|}{ (100) Lamellara) } \\
\hline & $\begin{array}{c}\text { distance } \\
[\AA]] \\
\end{array}$ & $\begin{array}{c}\text { FWHM }^{\mathrm{b})} \\
{\left[\AA^{-1}\right]}\end{array}$ & $\begin{array}{c}\mathrm{CCL}^{\mathrm{c})} \\
{[\AA]}\end{array}$ & $\begin{array}{c}\text { distance } \\
{[\AA ̊]} \\
\end{array}$ & $\begin{array}{c}\text { FWHM }^{\mathrm{b})} \\
{\left[\AA^{-1}\right]}\end{array}$ & $\begin{array}{c}\mathrm{CCL}^{\mathrm{c})} \\
{[\AA \AA]} \\
\end{array}$ \\
\hline $\begin{array}{l}\text { PNDI- } \\
\text { DTBT }\end{array}$ & 3.74 & 0.336 & 18.69 & 24.93 & 0.047 & 133.62 \\
\hline PBDB-T & 3.61 & 0.195 & 32.21 & 26.17 & 0.050 & 125.60 \\
\hline $\mathrm{J} 51$ & 3.55 & 0.275 & 22.84 & 22.76 & 0.066 & 95.15 \\
\hline PCE10 & 3.86 & 0.400 & 15.70 & 20.94 & 0.115 & 54.61 \\
\hline $\begin{array}{l}\text { PBDB- } \\
\mathrm{T}: \mathrm{A}^{\mathrm{d}}\end{array}$ & 3.60 & 0.265 & 23.70 & $\sim 24.15$ & 0.094 & 66.81 \\
\hline J51:A & 3.57 & 0.231 & 27.19 & $\sim 25.53$ & 0.059 & 106.44 \\
\hline PCE10:A & 3.85 & 0.401 & 15.66 & $\sim 21.22 / 23.31$ & $0.032 / 0.122$ & $196.35 / 51.50$ \\
\hline
\end{tabular}

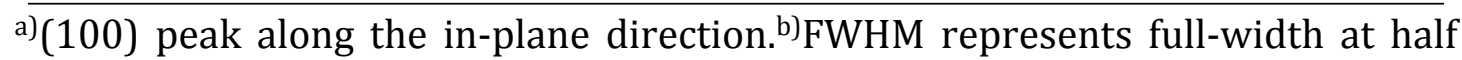
maximum. ${ }^{\text {c)} C C L ~ r e p r e s e n t s ~ c r y s t a l l i t e ~ c o h e r e n c e ~ l e n g t h . ~ d) ~ T h e ~ A ~ r e p r e s e n t s ~ f o r ~}$ the acceptor PNDI-DTBT.
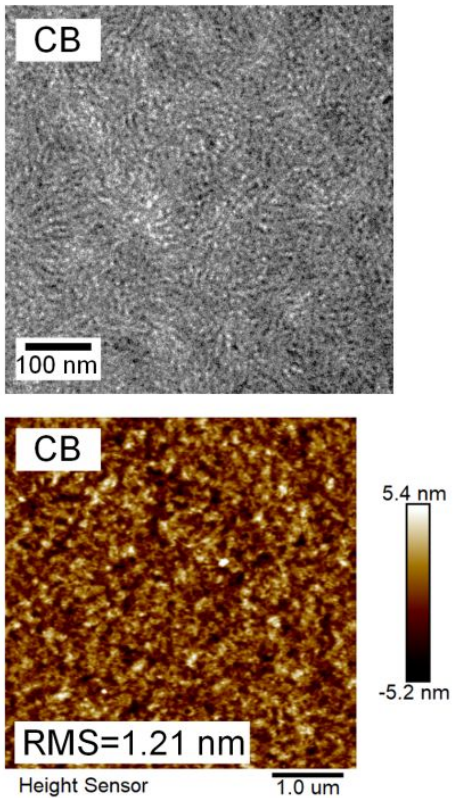
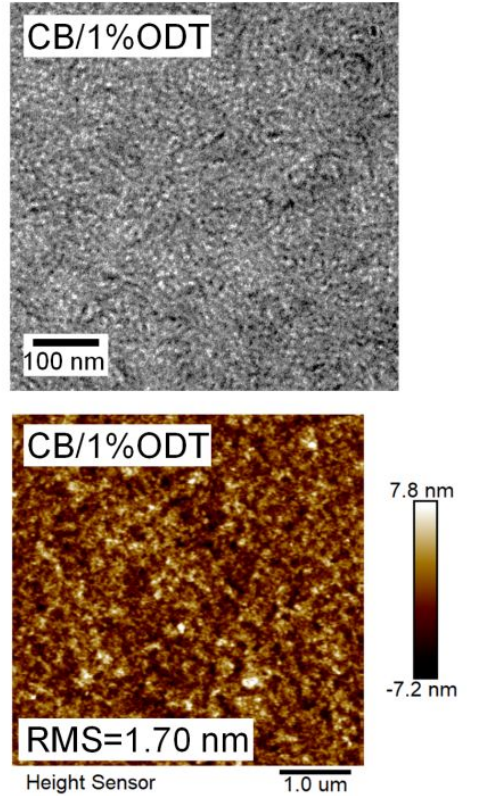
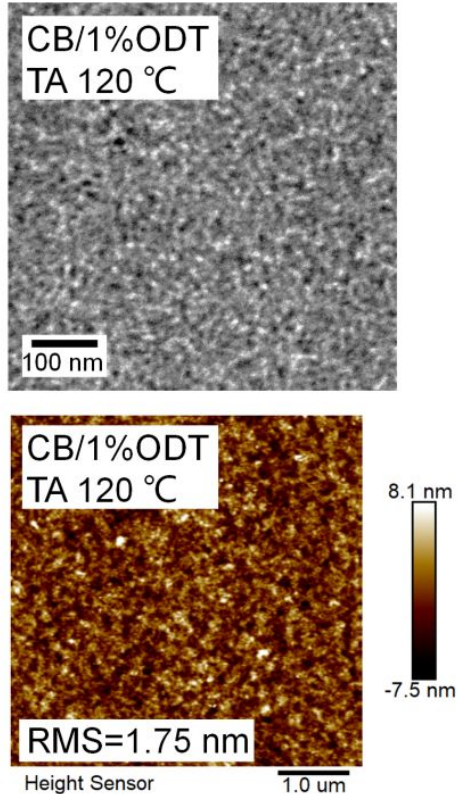

Figure S8. Morphology of PBDB-T:PNDI-DTBT under different processing conditions: TEM images (upper row), and AFM height images (lower row). 

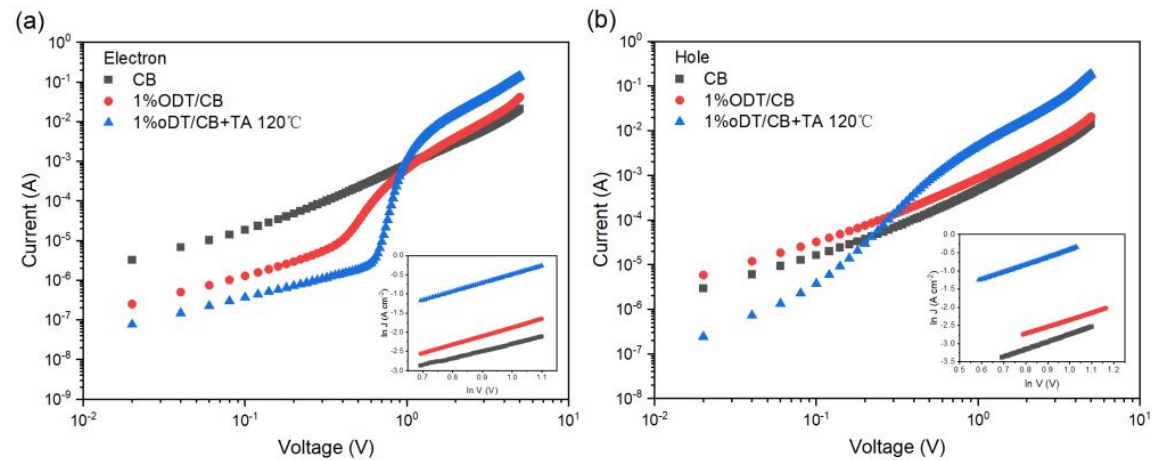

Figure S9. The current density versus voltage characteristics of PBDB-T:PNDIDTBT under different processing conditions. (a) electron-only devices with a structure of ITO/ZnO/active layer/PFN-Br/Ag, and (b) hole-only devices with a structure of ITO/PEDOT:PSS/active layer $/ \mathrm{MoO}_{3} / \mathrm{Ag}$. The insets show the fitting region.

Table S13. Charge carrier mobilities of PBDB-T:PNDI-DTBT under different processing conditions.

\begin{tabular}{lcccc}
\hline $\begin{array}{l}\text { Solvent } \\
{[\mathrm{v} / \mathrm{v}]}\end{array}$ & Annealing & $\begin{array}{c}\mu_{\mathrm{e}} \\
{\left[\mathrm{cm}^{2} \mathrm{~V}^{-1} \mathrm{~s}^{-1}\right]}\end{array}$ & $\begin{array}{c}\mu_{\mathrm{h}} \\
{\left[\mathrm{cm}^{2} \mathrm{~V}^{-1} \mathrm{~s}^{-1}\right]}\end{array}$ & $\mu_{\mathrm{e}} / \mu_{\mathrm{h}}$ \\
\hline $\mathrm{CB}$ & $\mathrm{w} / \mathrm{o}$ & $2.2 \times 10^{-4}$ & $1.5 \times 10^{-4}$ & 1.47 \\
$\mathrm{CB} / 1 \%$ ODT & $\mathrm{w} / \mathrm{o}$ & $3.4 \times 10^{-4}$ & $2.2 \times 10^{-4}$ & 1.54 \\
$\mathrm{CB} / 1 \%$ ODT & $120^{\circ} \mathrm{C}, 10 \mathrm{~min}$ & $1.4 \times 10^{-3}$ & $9.9 \times 10^{-4}$ & 1.41 \\
\hline
\end{tabular}

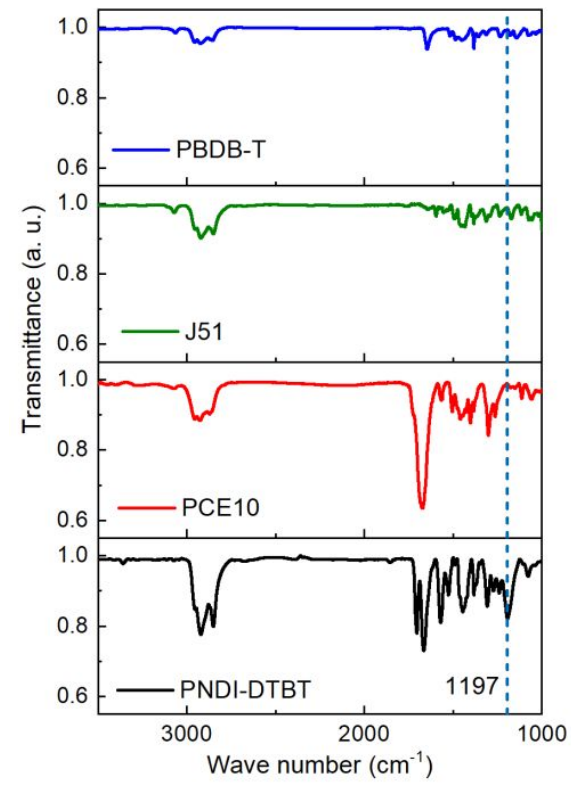

Figure S10. FT-IR spectra of PBDB-T, J51, PCE10, and PNDI-DTBT. 

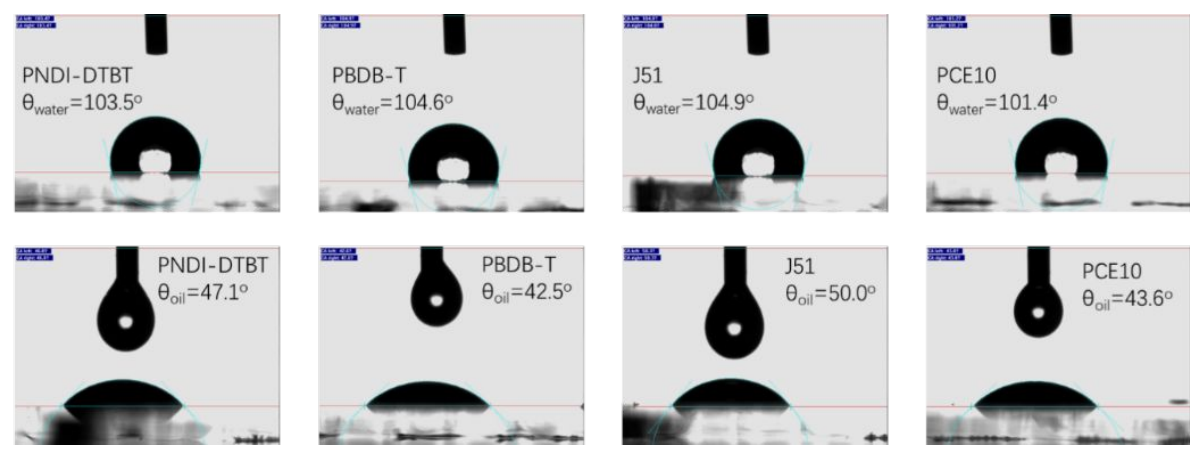

Figure S11. Views of surface contact measurements with different films by using deionized water (upper row) and diiodomethane (lower row) as the wetting liquid.

Table S14. The contact angles and surface energy parameters of the films.

\begin{tabular}{|c|c|c|c|c|c|}
\hline \multirow{2}{*}{ Film } & \multicolumn{2}{|c|}{ Contact angle } & \multirow{2}{*}{$\begin{array}{c}\gamma_{\mathrm{S}}^{\mathrm{D}} \\
{\left[\mathrm{mN} \mathrm{m}^{-1}\right]}\end{array}$} & \multirow{2}{*}{$\begin{array}{c}\gamma_{\mathrm{S}}^{\mathrm{P}} \\
{\left[\mathrm{mN} \mathrm{m}^{-1}\right]}\end{array}$} & \multirow{2}{*}{$\begin{array}{c}\text { surface energy } \\
\gamma_{S}\left[\mathrm{mN} \mathrm{m}^{-1}\right]\end{array}$} \\
\hline & $\theta_{\text {wat }}{ }^{\mathrm{a})}\left[{ }^{0}\right]$ & $\theta_{\text {oil }}{ }^{\text {b) }}\left[{ }^{\circ}\right]$ & & & \\
\hline PNDI-DTBT & 103.5 & 47.1 & 38.49 & 0.71 & 39.20 \\
\hline PBDB-T & 104.6 & 42.5 & 41.71 & 1.33 & 43.04 \\
\hline J51 & 104.9 & 50.0 & 36.85 & 0.75 & 37.60 \\
\hline PCE10 & 101.4 & 43.6 & 40.30 & 0.58 & 40.88 \\
\hline
\end{tabular}

${ }^{\text {a) }} \theta_{\text {oil }}$ represents the contact angle of deionized water. b) $\theta_{\text {oil }}$ represents the contact angle of diiodomethane.
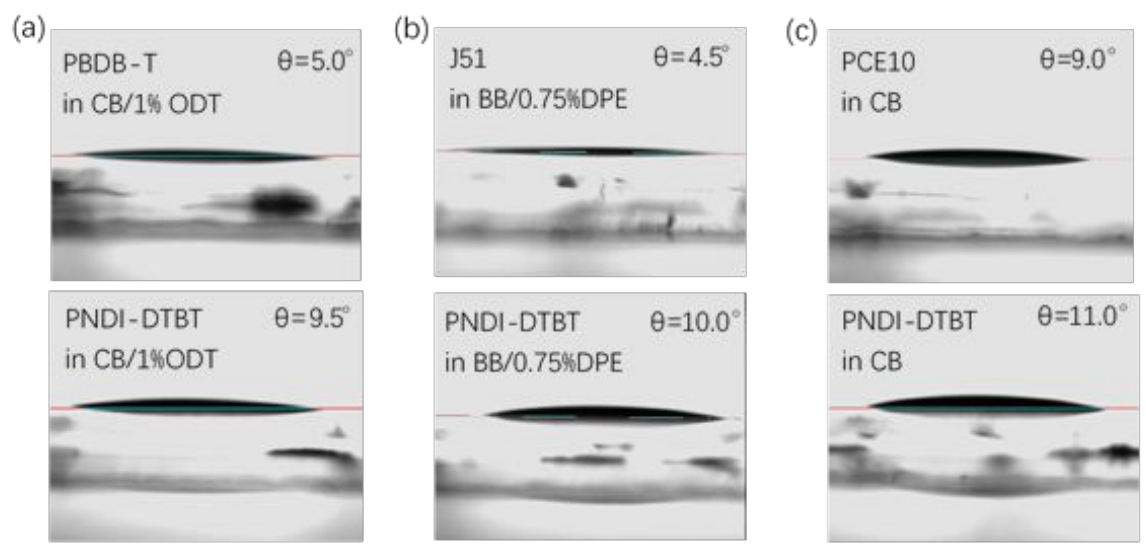

Figure S12. Views of surface contact measurements of different polymer solutions on PEDOT:PSS film: (a) PBDB-T and PNDI-DTBT, (b) J51 and PNDI-DTBT, (c) PCE10 and PNDI-DTBT. The same solvents for dissolving the donor:acceptor blends in all-PPDs fabrication were employed for each polymer in these measurements. 
Table S15. The surface tension and spreading parameters of the polymers on PEDOT:PSS.

\begin{tabular}{|c|c|c|c|c|}
\hline Polymer & $\begin{array}{c}\text { face energy o } \\
\text { liquid } \\
\gamma_{\mathrm{L}}\left[\mathrm{mN} \mathrm{m}^{-1}\right]\end{array}$ & $\begin{array}{c}\text { Contact angle } \\
\theta\left[^{\circ}\right]\end{array}$ & $\begin{array}{c}\Delta W \\
{[\mathrm{mN} \mathrm{m-1]}}\end{array}$ & $\begin{array}{l}\Delta W_{\mathrm{D}}-\Delta W_{\mathrm{A}} \\
{[\mathrm{mN} \mathrm{m}-1]}\end{array}$ \\
\hline PBDB-T & 20.2 & 5.0 & -0.1 & \multirow{2}{*}{0.2} \\
\hline PNDI-DTBTa) & 21.4 & 9.5 & -0.3 & \\
\hline $\mathrm{J} 51^{\mathrm{b})}$ & 21.4 & 4.5 & -0.1 & \multirow{2}{*}{0.3} \\
\hline PNDI-DTBT ${ }^{\mathrm{b}}$ ) & 27.2 & 10.0 & -0.4 & \\
\hline PCE10 & 21.1 & 9.0 & -0.3 & \multirow{2}{*}{0.1} \\
\hline PNDI-DTBTc) & 22.7 & 11.0 & -0.4 & \\
\hline \multicolumn{5}{|c|}{$\begin{array}{l}\text { a-c)The same solvents for dissolving the donor:acceptor blends in all-PPDs } \\
\text { fabrication were employed for each polymer in these measurements: }{ }^{\text {a)PBDB-T }} \\
\text { and PNDI-DTBT were dissolved in a solvent mixture of chlorobenzene with } 1 \% \\
\text { 1,8-octanedithiol in volume; b)J } 51 \text { and PNDI-DTBT were dissolved in a solvent } \\
\text { mixture of bromobenzene with } 0.75 \% \text { diphenyl ether in volume; }{ }^{c} \text { PCE10 and } \\
\text { PNDI-DTBT were dissolved in chlorobenzene. }\end{array}$} \\
\hline
\end{tabular}

\section{References}

(1) Zhao, Z.; Yin, Z.; Chen, H.; Zheng, L.; Zhu, C.; Zhang, L.; Tan, S.; Wang, H.; Guo, Y.; Tang, Q.; Liu, Y. High-Performance, Air-Stable Field-Effect Transistors Based on Heteroatom-Substituted Naphthalenediimide-Benzothiadiazole Copolymers Exhibiting Ultrahigh Electron Mobility up to $8.5 \mathrm{~cm} \mathrm{~V}^{-1} \mathrm{~s}^{-1}$. Adv. Mater. 2017, 29, 1602410.

(2) Zhuang, W.; Bolognesi, M.; Seri, M.; Henriksson, P.; Gedefaw, D.; Kroon, R.; Jarvid, M.; Lundin, A.; Wang, E.; Muccini, M.; Andersson, M. R. Influence of Incorporating Different Electron-Rich Thiophene-Based Units on the Photovoltaic Properties of Isoindigo-Based Conjugated Polymers: An Experimental and DFT Study. Macromolecules 2013, 46, 8488-8499.

(3) Steckler, T. T.; Henriksson, P.; Mollinger, S.; Lundin, A.; Salleo, A.; Andersson, M. R. Very Low Band Gap Thiadiazoloquinoxaline Donor-Acceptor Polymers as Multi-tool Conjugated Polymers. J. Am. Chem. Soc. 2014, 4, 1190-1193.

(4) Leenaers, P. J.; van. Eersel, H.; Li, J.; Wienk, M. M.; Janssen. R. A. J. Influence of Regioregularity on the Optoelectronic Properties of Conjugated Diketopyrrolopyrrole Polymers Comprising Asymmetric Monomers. Macromolecules 2020, 53, 7749-7758.

(5) Bin, H.; Wang, J.; Li, J.; Wienk, M. M.; Janssen, R. A. J. Efficient Electron Transport Layer Free Small-Molecule Organic Solar Cells with Superior Device Stability. Adv. Mater. 2021, 33, 2008429. 
(6) van Franeker, J. J.; Hermida-Merino, D.; Gommes, C.; Arapov, K.; Michels, J. J.; Janssen, R. A. J.; Portale, G. Sub-Micrometer Structure Formation during Spin Coating Revealed by Time-Resolved In Situ Laser and X-Ray Scattering. Adv. Funct. Mater. 2017, 27, 1702516.

(7) Kouijzer, S.; Michels, J. J.; van den Berg, M.; Gevaerts, V. S.; Turbiez, M.; Wienk, M. M.; Janssen, R. A. J. Predicting Morphologies of Solution Processed Polymer:Fullerene Blends. J. Am. Chem. Soc. 2013, 135, 12057-12067.

(8) Nilsson, S.; Bernasik, A.; Budkowski, A.; Moons, E. Morphology and Phase Segregation of Spin-Casted Films of Polyfluorene/PCBM Blends. Macromolecules 2007, 40, 8291-8301.

(9) Flory, P. J., Principles of Polymer Chemistry. Cornell University Press: Ithaca, NY, USA, 1953.

(10) de Gennes, P. G. Wetting: statics and dynamics. Rev. Mod. Phys. 1985, 57, $827-$ 863.

(11) Wöbkenberg, P. H.; Ball, J.; Kooistra, F. B.; Hummelen, J. C.; de Leeuw, D. M.; Bradley, D. D. C.; Anthopoulos, T. D. Low-voltage organic transistors based on solution processed semiconductors and self-assembled monolayer gate dielectrics. Appl. Phys. Lett. 2008, 93, 013303.

(12) Zhang, L.; Yang, T.; Shen, L.; Fang, Y.; Dang, L.; Zhou, N.; Guo, X.; Hong, Z.; Yang, Y.; Wu, H.; Huang, J.; Liang, Y. Toward Highly Sensitive Polymer Photodetectors by Molecular Engineering. Adv. Mater. 2015, 27, 6496-503.

(13) Zhang, J.; Kwok, D. Y. Calculation of Solid-Liquid Work of Adhesion Patterns from Combining Rules for Intermolecular Potentials. J. Phys. Chem. B 2002, 106, 12594-12599. 